\title{
INVESTIGATION OF THE EXPERIMENTAL TANDEM-X PURSUIT MONOSTATIC MODE FOR OIL AND SHIP DETECTION
}

\author{
Domenico Velotto ${ }^{1}$, Ferdinando Nunziata ${ }^{2}$, Carlos Bentes ${ }^{1}$, Maurizio Migliaccio $^{2}$ and Susanne Lehner ${ }^{1}$ \\ ${ }^{1}$ DLR, German Aerospace Center, Remote Sensing Technology Institute \\ 82234 Bremen, Germany \\ ${ }^{2}$ Università di Napoli Parthenope, Dipartimento di Ingegneria \\ 80143 Napoli, Italy
}

\begin{abstract}
The German Aerospace Center has opened the TanDEM-X science phase in order to demonstrate new Synthetic Aperture Radar techniques and applications. In particular, the TanDEM-X pursuit monostatic configuration has been switched on from October 2014 till February 2015.

This paper investigates the use of the two TanDEM-X satellites to acquire a pair of independent SAR data in single and multi-polarization mode for maritime surveillance applications. Multi-polarization SAR data have shown to be more effective than singlepolarization one, for what concern oil spill and ship detection. Here, for the first time has been possible to directly compare almost simultaneous SAR measurements of marine slicks and targets using both single and multi-polarization data. In-situ ground truth data have been collected during satellites overpasses in order to conduct the analysis.
\end{abstract}

Index Terms - Pursuit monostatic radars, SAR, oil spill, ship detection, PolSAR, Dual Receive Antenna

\section{INTRODUCTION}

In the recent years the scientific community has addressed and proved the importance of Polarimetric SAR (PolSAR) data in the many oceanographic applications, e.g. ship detection, oil spill, ice type classification, coastline extraction, etc. In this paper the focus is on the first two applications: oil and ship detection. Although, upon a physical interpretation of the polarimetric information, the methods proposed in literature have shown that dual- and quad-pol data are preferred over single-pol data [1]-[4], the drawbacks of PolSAR configuration haven't been taken into account. As matter of fact, due to the technological limitations of the current spaceborne SAR able of PolSAR acquisitions, the gained information is paid at the cost of worse resolution and less across-track coverage. This is because the current radars are pulsed radar, which can transmit alternatively pulses with different polarisation state, i.e. Horizontal $(\mathrm{H})$ or Vertical (V), and receive alternatively in $\mathrm{H}$ or $\mathrm{V}$ polarisation, forming the 4 channels $\mathrm{HH}, \mathrm{HV}, \mathrm{VH}$ and VV. Compared to single-pol acquisition, where only one polarization state is transmitted and only one is received at pulse repetition frequency (PRF), for PolSAR acquisitions the total PRF must be doubled. As consequence the Doppler bandwidth per channel is reduced, with causes a loss in azimuth resolution, and the ambiguous range region increase, which causes the across-track swath to be narrowed down. Many polarimetric oil and ship detectors have been developed and all of them share the common result that PolSAR data increase the probability of detection assuming the same false alarm rate. Usually the performances of PolSAR detectors can only be directly compared with the ones obtainable using one of the channels. Although this comparison strategy is quite fair, it has never been possible to compare PolSAR acquisitions with single-pol acquisitions, e.g. PolSAR at lower resolution vs single-pol at full resolution. It is obvious that such comparison can only be done if the acquisitions are simultaneously (or close to be simultaneous) made by two twins sensors. For this analysis we have exploited the pursuit monostatic configuration of the TanDEM-X, an Xband SAR constellation composed of two satellites flying in close formation. In such configuration the twin satellites TerraSAR-X (TS-X) and TanDEM-X (TD-X) are flying in two orbits separated of circa $76 \mathrm{~km}$ (that would correspond to approximately $10 \mathrm{sec}$ ). 


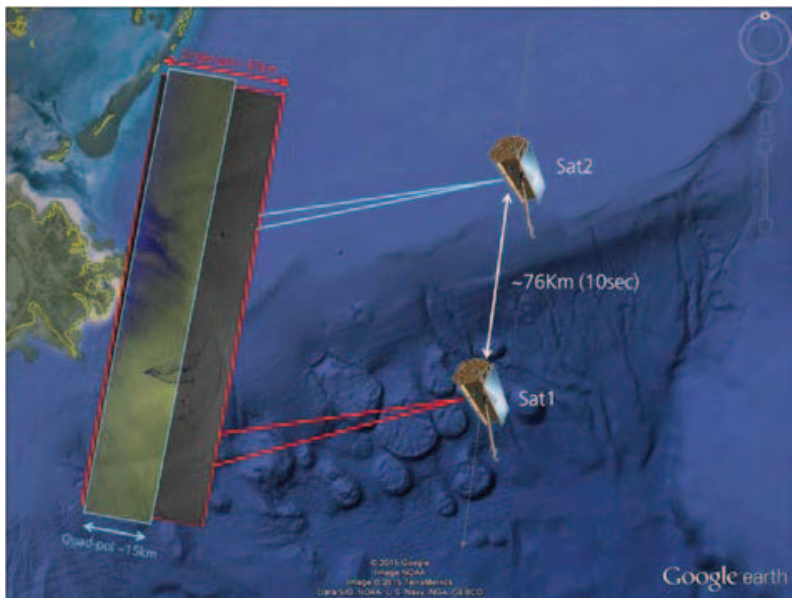

Figure 1. Artists view of the TD-X pursuit monostatic configuration combined with SRA single-pol (fore platform Sat1, in red the swath coverage) and DRA PolSAR (aft platform Sat2, in cyan the swath coverage) SM imaging modes.

Moreover, on both satellites is possible to set-up the Dual Receive Antenna (DRA) configuration, i.e. electronically splitting of the antenna in two halves in azimuth direction to emulate two complete receiving channels. In this way, it has been possible to further exploit the TD-X flexibility and acquire two independent single-pol and quad-pol dataset (see Figure 1).

\section{OIL SPILL}

In this time frame, the spot that has been selected for such experiment is an oil field located in the North part of the Gulf of Mexico near the Louisiana river delta. This area is well known because of the environmental disaster caused by the BP Deep Water Horizon platform blowout. Since the accident, the area has been continuously monitored by TS-X and a persistent spill, not connected to any platform in the area, has been found not far from the Deep Water Horizon platform. The persistent spill is actually due to a well that had been damaged by Hurricane Ivan in 2004 and have presumably been leaking steadily ever since. Furthermore, the area is interesting for the high density of metallic platforms and ships.

Figure 2 shows the sub-scenes extracted from the SAR data acquired by Sat1 in single-pol (HH polarization, top left) and Sat2 in quad-pol (HH polarization displayed only, top right) where an extended spill is clearly visible as dark area. The plots at the bottom of Figure 2 are the slick-free and slick-covered radar
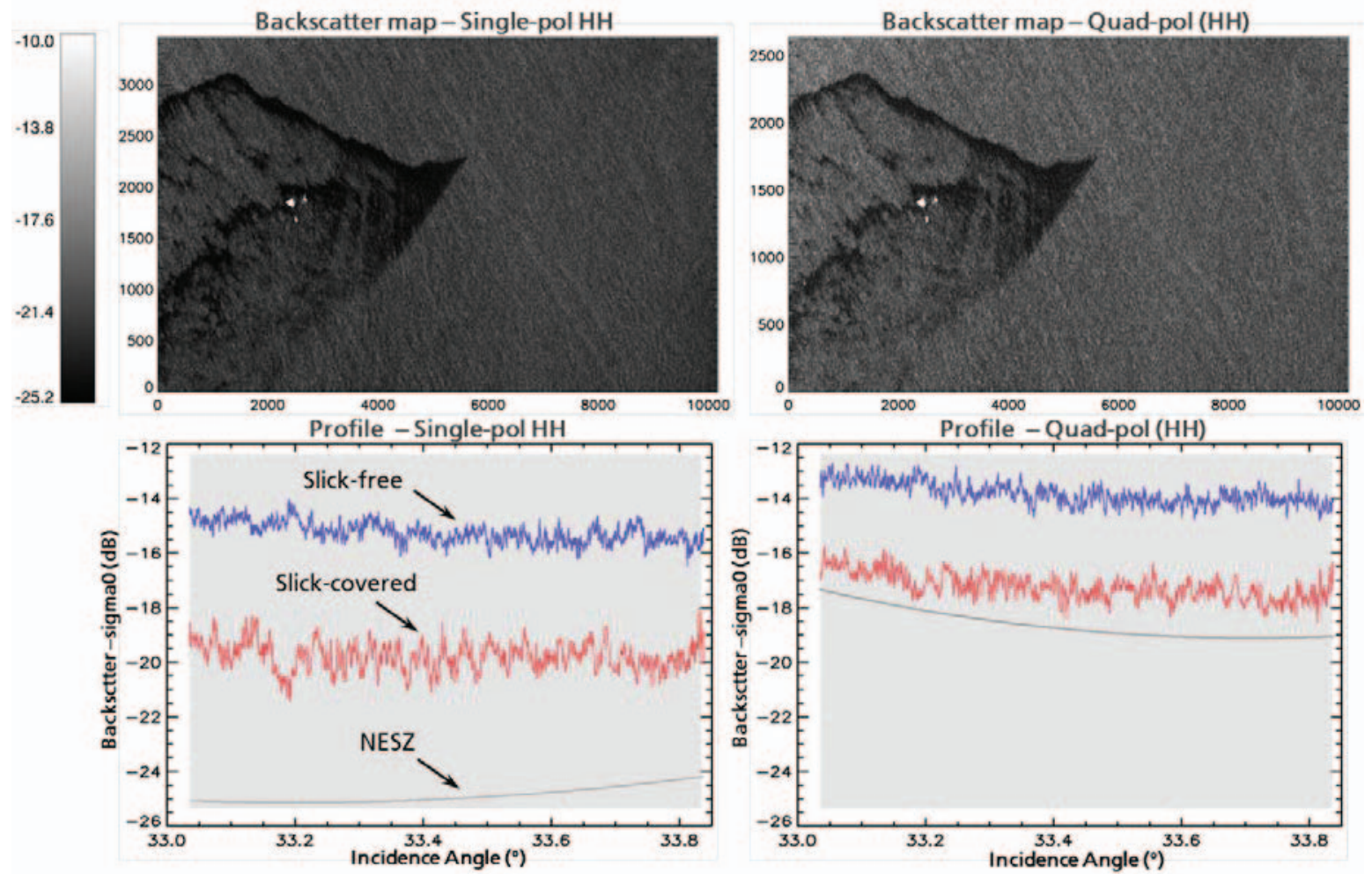

Figure 2. Oil spill backscatter analysis and comparison with annotated NESZ for single- and quad-pol acquired data. 

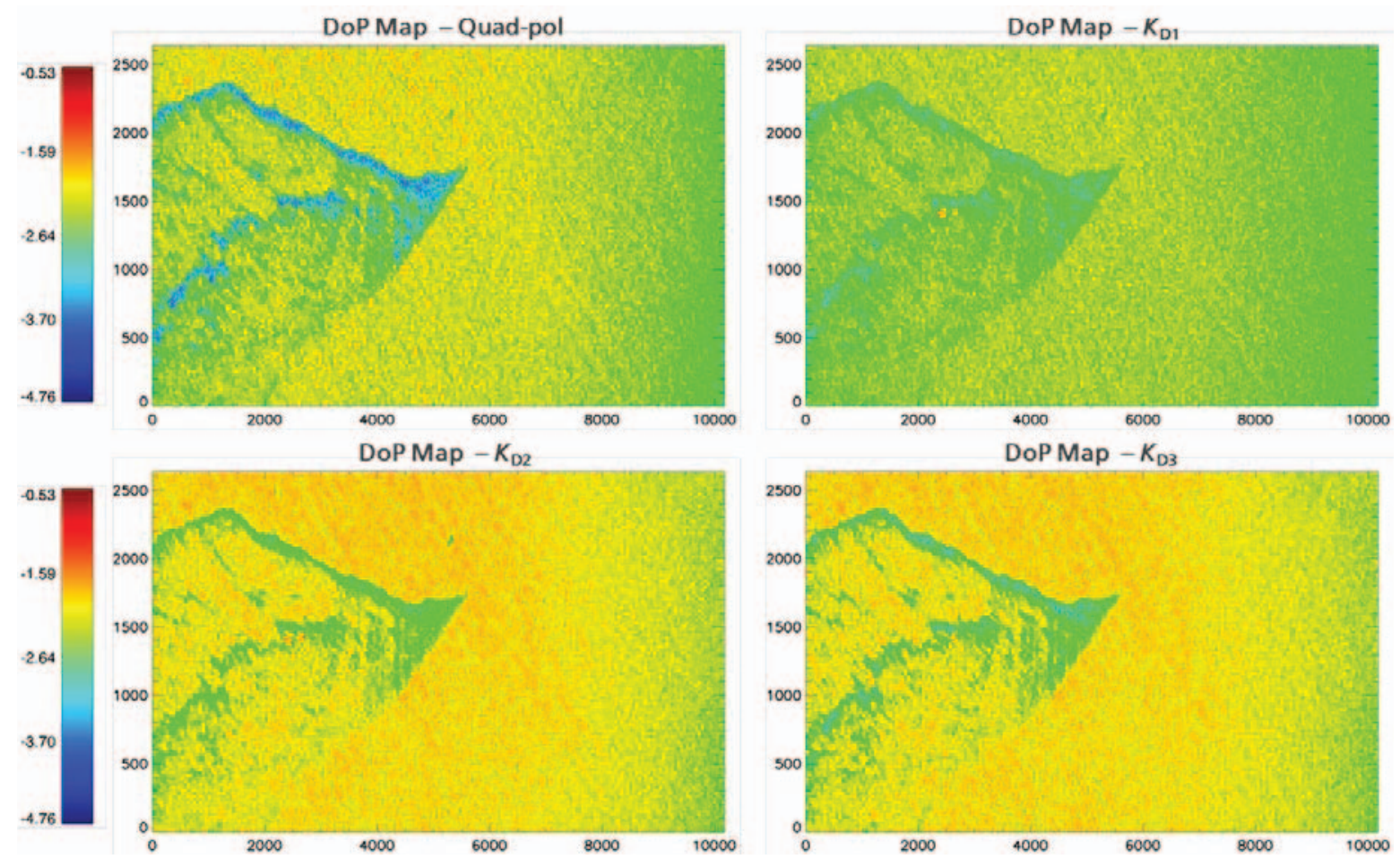

Figure 3. Oil spill Degree of Polarization comparison between quad-pol and the three combination of dual-pol possible, i.e $k_{D 1}=\left[\dot{S}_{h h}, \dot{S}_{h v}\right]^{T}, k_{D 2}=\left[\dot{S}_{v h}, \dot{S}_{v v}\right]^{T}$ and $k_{D 3}=\left[\dot{S}_{h h}, \dot{S}_{v v}\right]^{T}$.

backscatter profiles extracted for single-pol $\mathrm{HH}$ (bottom left) and quad-pol HH (bottom right). These plots demonstrate that for both cases, slick-free and slick-covered signal are above the annotated noise Equivalent Sigma Zero (NESZ).

In [5] the Degree of Polarization (DoP), defined in terms of the elements of the Muller matrix $M$, has been used has an efficient parameters for oil slicks observation using quad-pol C-band SAR data.

In [6] the performance of the DoP is studied for joint ship and oil-spill detection under different polarizations in hybrid/compact and linear dual-pol SAR airborne L-band and space-borne C-band data. The DoP in [6] is estimated starting from the definition of the Stokes parameters. In this case, differently from the definition in terms of $M$, dual-pol data are sufficient.

For the purposes of this investigation, the DoP retrieved using $M$ (hence linear quad-pol data) and using Stokes parameters (here limited to linear dualpol data) are used and compared regarding oil spill observation. The result of such comparison is shown in Figure 3. From these preliminary results, it is deducible that DoP estimation exploiting quad-pol data shows higher sensitivity to oil spill thickness. The closest result using linear dual-pol are obtained using the combination HH-VV, agreeing with the performance analysis in [6].

\section{SHIPS AND MARITIME TARGETS}

In order to evaluate the performances and benefits of the proposed new imaging and polarimetric arrangement for ship and maritime targets applications, several data-take orders have been planned and successfully acquired over ship traffic lane and known static marine targets.

The example proposed in Figure 4, shows a moving ship (reported AIS info are: length $177 \mathrm{~m}$, breadth $29 \mathrm{~m}$ and speed $7.4 \mathrm{kn}$ ) imaged by the fore platform $($ Sat1=TS-X) in StripMap single-pol VV $(1.2 \times 3.3 \mathrm{~m}$ nominal resolution, Figure 4(a)) and the aft platform (Sat2=TD-X) in StripMap dual-pol HH-VV (1.2 x $6.6 \mathrm{~m}$ nominal resolution, Figure $4(\mathrm{~b})$ and (c), respectively). Figure $4(\mathrm{~d})$ shows the composite of the fore platform (TS-X) and aft platform (TD-X) datatakes in VV polarization. The loss in azimuth resolution, due to the dual-pol acquisition, does not change considerably the radar signal received from the ship structures. This is further shown by the ship 


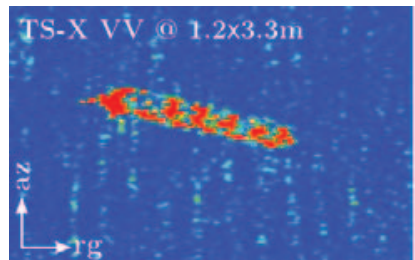

(a)

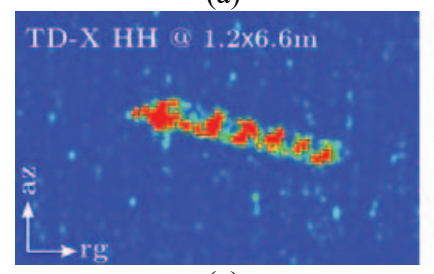

(c)

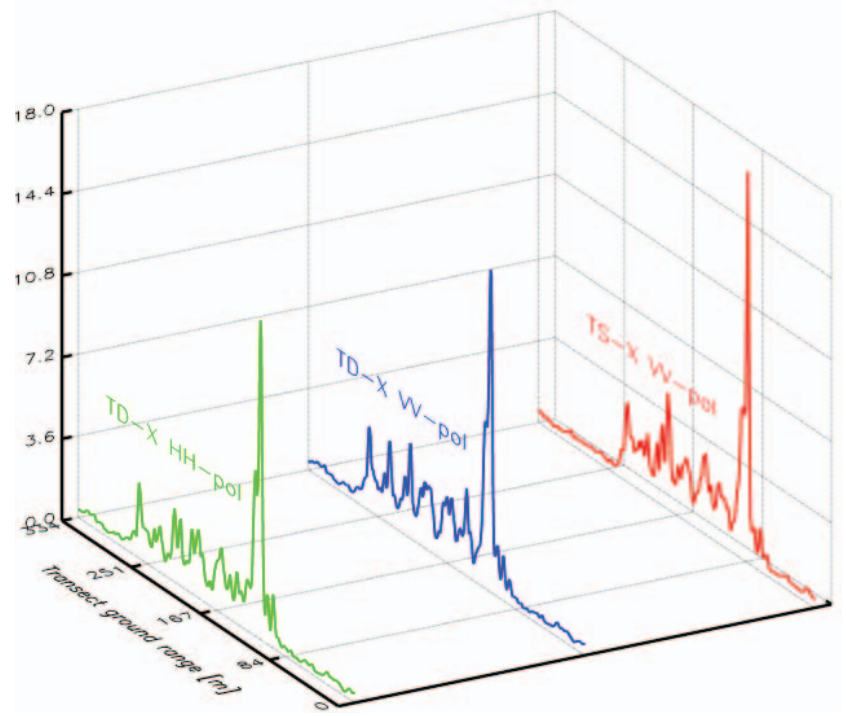

(e)

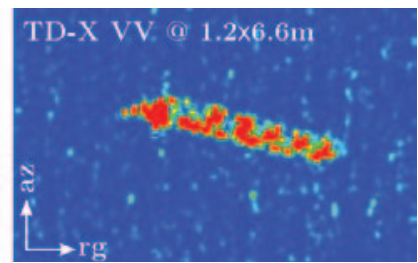

(b)

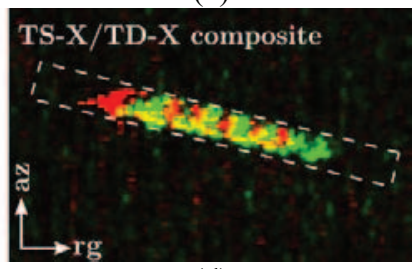

(d) applications. System noise, polarimetric capabilities of different polarization combination and resolution are design parameters that have been took into account.

\section{REFERENCES}

[1] M. Migliaccio, A. Gambardella, and M. Tranfaglia, "SAR polarimetry to observe oil spills," IEEE Trans. Geosci. Remote Sens., vol. 45, no. 2, pp. 506-511, 2007.

[2] D. Velotto, M. Migliaccio, F. Nunziata, and S. Lehner, "Dual-polarized TerraSAR-X data for oil-spill observation," Geosci. Remote Sens. IEEE Trans. On, vol. 49, no. 12, pp. 4751-4762, 2011.

[3] D. Velotto, M. Soccorsi, and S. Lehner, "Azimuth Ambiguities Removal for Ship Detection Using Full Polarimetric X-Band SAR Data," IEEE Trans. Geosci. Remote Sens., vol. 52, no. 1, pp. 76-88, Jan. 2014.

[4] A. Marino, "A Notch Filter for Ship Detection With Polarimetric SAR Data," IEEE J. Sel. Top. Appl. Earth Obs. Remote Sens., vol. 6, no. 3, pp. 1219-1232, Jun. 2013.

[5] F. Nunziata, A. Gambardella, and M. Migliaccio, "On the degree of polarization for SAR sea oil slick observation," ISPRS J. Photogramm. Remote Sens., vol. 78, pp. 41-49, Apr. 2013.

[6] R. Shirvany, M. Chabert, and J.-Y. Tourneret, "Ship and OilSpill Detection Using the Degree of Polarization in Linear and Hybrid/Compact Dual-Pol SAR," IEEE J. Sel. Top. Appl. Earth Obs. Remote Sens., vol. 5, no. 3, pp. 885-892, Jun. 2012.

[7] S. V. Baumgartner and G. Krieger, "Large along-track baseline SAR-GMTI: First results with the TerraSAR$\mathrm{X} /$ TanDEM-X satellite constellation," in Geoscience and Remote Sensing Symposium (IGARSS), 2011 IEEE International, 2011, pp. 1319-1322.
Figure 4. Cargo ship imaged by TD-X satellites in pursuit monostatic configuration (Sat1 in single-pol VV and Sat2 in dual-pol HH-VV)

signature profiles (along the main axis) in Figure 4(e). The one dimensional cross-correlation analysis between the retrieved ship's profiles, leads to a travelled distance of $39 \mathrm{~m}$, which corresponds to an average speed of $7.6 \mathrm{kn}$ (temporal baseline 10 seconds). This very preliminary ship speed accuracy analysis of the proposed imaging arrangement, is in line with the performances of the classic TD-X dualplatform SAR-GMTI demonstrated in [7].

\section{CONCLUSIONS}

In this paper, oil spill and ship detection are investigated by using TD-X pursuit monostatic SAR data. A new arrangement of imaging and polarization arrangement is proposed and evaluated for these 